\title{
viewpoint
}

\section{Tragic and sudden death}

\author{
Potential and proven mechanisms causing sudden infant death syndrome
}

\author{
Bradley Thach
}

$\mathrm{T}$ he death of a child is probably the most traumatic event any parent can experience. This tragedy is made worse if the child dies in its sleep, apparently for no reason. Sudden infant death syndrome (SIDS) inevitably strikes horror into parents' hearts; it usually happens at night and occurs in complete silence-there is no fight for life or breath to warn parents that something is horribly wrong. Indeed, infants who have died of SIDS look rather peaceful, which makes it even worse for parents who wake up in the morning to find their child dead.

\section{SIDS $[\ldots]$ continues to thwart the efforts of scientists and physicians to prevent it, or at least to identify those children who are at risk}

SIDS is not only a traumatic and devastating event for parents and medical examiners, it is also a cause of death that continues to thwart the efforts of scientists and physicians to prevent it, or at least to identify those children who are at risk. Indeed, the term SIDS was coined to provide a convenient diagnosis for a medical examiner to label an otherwise unexplainable death.

It is only during the past few years that scientists and epidemiologists have been able to identify environmental and metabolic factors that combine to cause SIDS. In particular, epidemiological research has identified some important environmental risk factors-notably when children sleep on their stomachs or under heavy blankets, or whether the parents smoke. One resulting recommendation is to encourage parents to turn infants on their backs; the 'Back to
Sleep' campaign that started in 1994 in the USA and similar educational campaigns in other countries have led to a considerable drop in SIDS deaths. Nevertheless, the prevalence of SIDS is still approximately $0.5 \%$ in the USA, or 50 deaths per 100,000 live births, and it still affects children who sleep on their backs. Therefore, the main objective of the ongoing research into the genetics, physiology and epidemiology of SIDS is to identify more risk factors.

In any case, SIDS is not a uniquely 'identifiable disease'; it is a diagnosis of exclusion that should only be applied to the death of an infant after an adequate post-mortem investigation-including an autopsy, a mandatory investigation of the death scene and an exploration of a possible family history-has provided no explanation. Indeed, the term 'sudden unexplained death in infants' is being increasingly used as it becomes evident that a number of deaths that were formerly termed SIDS were actually accidental suffocation, homicide, cardiac arrhythmias or inborn errors of metabolism.

S IDS is a combination of genetic, metabolic and environmental factors that culminate in the death of an infant. Not surprisingly, the literature abounds with many proposed mechanisms, theories and scientific concepts, which are rapidly changing. Nonetheless, researchers have been able to identify various prenatal and postnatal factors that increase the risk of SIDS (Sidebar A).

The greatest advances in elucidating the causes of risk for SIDS and formulating preventive measures have come through mandatory death scene investigations. In many cases, these often indicate that the airway of the child was compromised at the time of death. As further research establishes the pathophysiology of various types of infant suffocation, the number of diagnoses of 'accidental suffocation', 'wedging' or 'positional asphyxia' have increased, whereas the formerly preferred diagnosis, SIDS, is now used less frequently. Unger and colleagues estimate that up to $30 \%$ of sudden unexplained deaths in infants can be attributed to accidental suffocation (Unger et al, 2003). Therefore, in recent years, there has been much attention paid to the various mechanisms that lead to accidental suffocation, including studies that have used mechanical models, animals and live infants to investigate how bedding obstructs air exchange.

W hen an infant's head is completely covered, or when it is lying face down into its bedding, exhaled air can be trapped to a varying extent (Unger et al, 2003; Patel et al, 2001). The child re-breathes the exhaled air, which, over time, leads to high levels of carbon dioxide and increasingly low levels of oxygen in the air pocket formed between the infant's head and the bedding. For infants who sleep face down, the softness of the bedding determines the depth of this air pocket-the deeper the pocket, the greater the seal around the face and the less likely the chance that there will be air channels to allow access to fresh air. For infants whose head is covered, the thickness of the bedding determines the size of the trapped pool of air. If the bedding beneath the face or over the head is thin, an increase in tidal volume might be sufficient to prevent asphyxia- a dangerous depletion of oxygen in the blood-whereas a single heavy blanket might be sufficient to cause asphyxia.

Another crucial factor is an infant's own protective response, which is learned by 
experience (Paluszynska et al, 2004). Most infants who normally sleep face down frequently experience re-breathing during a single night and learn to lift or turn their head to the side. Infants who have never slept prone often fail to turn their heads to the side. Although they might lift their heads, this lasts for only a few seconds and they then continue to re-breathe exhaled air once their heads drop down (Paluszynska et al, 2004).

\section{...the main objective of the ongoing research into the genetics, physiology and epidemiology of SIDS is to identify more risk factors and how they contribute to it}

Once in a re-breathing environment, the rate of progression is highly variable. Slight changes in head position might create new air channels in the bedding to allow access to fresh air. If the infant does not move the head, the progression of asphyxia is usually slow and occurs over several minutes or longer. Arousal is usually accompanied by a sharp decrease in respiratory frequency. When this happens, the oxygen saturation might drop rapidly to $85 \%$ or less. Once oxygen saturation reaches $30-40 \%$, the brain is depleted of oxygen, hypoxic coma occurs and arousal is no longer possible. Even autoresuscitation by gasping is ineffective when the infant's head is in a hypoxic environment.

A nother documented cause of death is the compromising of an infant's airway by a sleeping parent or sibling when bed sharing; in recent years such deaths have increased as bed sharing has become more popular and could explain as many as $50 \%$ of sudden unexpected infant deaths (Unger et al, 2003; Blair et al, 2006). The presumed mechanism of death is accidental suffocation by close contact with a co-sleeper. However, the actual cause of death in this situation is usually very difficult to prove.

As mentioned above, educational campaigns with the aim of preventing both prone sleeping and bed sharing have led to a significant decrease in the number of infants dying from suffocation. Nevertheless, SIDS still occurs in seemingly healthy children, even if they sleep on their backs. The main focus of research is therefore to identify other risk factors and to investigate possible pathophysiological mechanisms, as well as theories of how various factors contribute to and cause SIDS. A plausible mechanistic theory is essential to develop diagnostic tests to accurately identify children at risk and, eventually, to develop preventive or therapeutic measures (Leiter \& Böhm, 2007).

A nother suspected precursor to sudden death in infants is heat stress - extreme elevations in body temperature that can cause fatal heat stroke. The idea that heat stress itself can result in SIDS is based on data from countries where it is common to dress infants in many layers of clothing or to cover them with quilts during the winter (Mitchell et al, 1991; Fleming et al, 1996). Because the face is a main source of heat elimination, it has been suggested that infants who are face down can die from heat stress. Proponents of this theory believe that heat stress might not necessarily be associated with an actual elevation in body temperature.

However, several observations argue against heat stress as being a major cause of SIDS. As dangerous asphyxia can occur very soon after an infant turns its face into the bedding, it would seem that this might cause death before the heat stress could take effect. This, of course, does not eliminate the possibility that a combination of elevated body temperature and re-breathing might be more lethal than either factor alone. In the USA, SIDS rates do not increase during summer heat waves when the country witnesses a marked increase in heat-related deaths (Scheers-Masters et al, 2003), which indicates that heat stress is not a main cause of SIDS, at least in the USA. Finally, perhaps the greatest problem with the heat stress theory is that there has been no pathophysiological mechanism identified so far that could cause sudden death.

$\mathrm{T}$ he eventual cause of death in SIDS infants is a failure to breathe. However, this should not happen: humans, as well as many animals, have evolved highly effective emergency reflexes to spontaneously recover from severe hypoxia (Thach et al, 1991). If hypoxia turns into coma, cortical activity in the central nervous system and most brainstem-mediated reflexes cease to function. This triggers a set of emergency reflexes that eventually reinstate normal respiration by intermittent hypoxic gasps that, in the
Sidebar A | Factors that increase the risk of sudden infant death syndrome (SIDS)

Prenatal risks

Inadequate care

Inadequate nutrition

Smoking

Use of heroin

Subsequent births less than 1 year apart

Alcohol abuse

Obesity

Postnatal risks

Low birth weight

Exposure to tobacco smoke

Sleep positioning

Excess clothing and overheating

Excess bedding, soft sleep surface and stuffed animals

Gender (61\% of SIDS cases occur in males)

Age (highest from 2 to 4 months)

Premature birth

absence of compromised lung or cardiovascular function, provide enough oxygen for the body to reinstate normal function.

\section{SIDS is a combination of genetic, metabolic and environmental factors that culminate in the death of an infant}

Several studies have analysed recordings of cardiac and respiratory activity just prior to the death of infants who have died of SIDS and other causes (Poets et al, 1999; Sridhar et al, 2003; Meny et al, 1994). Of the 18 or so SIDS deaths analysed so far, there have been only two cases in which the child failed to breathe before the onset of hypoxic gasping - in these cases, the lack of breathing might have caused the hypoxia. These home-monitored recordings showed an abruptly slower heartbeat-bradycardia—just before the onset of apnea-the suspension of breathing. This suggests that the preceding hypoxia was severe and already present during the 15-second period captured on tape just before the bradycardia; it is not clear what caused the infant to become hypoxic. It is relevant that, compared with infants dying of other causes, these SIDS infants showed a decreased ability to transiently increase their heart rate or recover normal breathing as a result of hypoxic gasping (Sridhar et al, 2003), although it is still not clear why autoresuscitation failed. 
I nfants born prematurely are notoriously susceptible to episodes of apnea, which can be generally distinguished into two types. Awake apnea is attributed to acute hypoventilation associated with breath holding as a result of pain or emotional upset (Abu-Osba et al, 1982). Such spells can result in sudden severe hypoxia with secondary hypoxic apnea. However, once the body's emergency mechanisms kick in, the children autoresuscitate by gasping. Indeed, the hypoventilation associated with ordinary crying has many similarities to clinical breath holding, which is often preceded by crying. Breath holding during arousal from sleep is a common cause of oxygen desaturation in pre-term infants (Abu-Osba et al, 1982). Although SIDS is believed to occur when infants are sleeping, the potential role of brief arousal accompanied by breath holding with severe hypoxia and failed autoresuscitation cannot be excluded as a cause for some SIDS deaths.

The second main type of apnea occurs primarily during sleep. The most important of these are obstructive sleep apnea (OSA) and reflex apnea caused by laryngeal chemoreflexes (LCRs; Pickens et al, 1988; Davies et al, 1988). As the muscle tone of the body ordinarily relaxes during sleep, the soft tissues that make up the walls of the human throat can collapse, thereby causing OSA, which, in a mild form, is quite common. LCRs are stimulated by hypochloride or acid solutions from the stomach content that contact mucosal nerve endings at the entrance to the larynx. They consist of apnea, swallowing, laryngeal closure and coughing. Unlike OSA, apnea in premature infants predominantly consists of respiratory pauses associated with sporadic obstructed breaths. It is usually associated with swallowing during the spell, a characteristic of LCR apnea, but it is not known to happen during OSA (Pickens et al, 1988).

Studies of sleeping human infants, stimulated with water infused into the pharynx, have shown that repeated swallowing, apnea and airway obstruction occurs more often in pre-term infants (Pickens et al, 1988; Davies et al, 1988). Furthermore, several studies have found that apnea and bradycardia associated with swallowing and intermittent airway obstruction occur spontaneously during sleep in pre-term infants, even in the absence of any detectable stimulus (Pickens et al, 1988; Miller \& DiFiore, 1995). Apnea can also occur through regurgitation of the stomach content. This suggests that endogenous stimuli from accumulated pharyngeal secretions or regurgitated gastric fluid can elicit LCR responses in pre-term infants (Pickens et al, 1989). Apnea therefore remains a viable hypothesis for sudden death in infants. In fact, a few of the home recordings of SIDS cases indicate that apnea preceded death (Poets et al, 1999; Sridhar et al, 2003; Meny et al, 1994). The apnea hypothesis is also strengthened by the observation that its persistence is much stronger in pre-term infants who are at an especially high risk for SIDS (Hunt et al, 2004).

nfection-particularly with the respiratory syncytial virus (RSV) - is another factor that is strongly suspected to trigger the fatal course of events that lead to SIDS. RSV is known to cause severe LCR-induced apnea, and infected infants occasionally require mechanical ventilation to prevent death. Furthermore, infants between two and four months of age actually have a significantly decreased number of red blood cells, which can greatly prolong LCR apnea (Fagenholz et al, 1979). Together, these facts suggest that viral infection with RSV and other viruses at the peak of prematurity anaemia could cause SIDS.

\section{It seems that most SIDS infants had multiple abnormalities in brain serotonergic systems, which could provide an explanation for why they were more vulnerable to death}

In support of the argument that viral infection could be a precursor for at least some cases of SIDS, Vege \& Rognum (2004) reported increased levels of interleukins in the cerebrospinal fluid of many SIDS victims. Other studies associated RSV infection with increased LCR reflexes and an increase in inflammatory interleukin in the laryngeal mucosa. It remains to be seen how increased interleukin in the brainstem might cause sudden death, but these observations support the theory of subtle infections as an initial cause of apnea and eventually SIDS. There is also epidemiological support for this theory: seasonal outbreaks of RSV during the winter months coincide with a peak of SIDS cases. Together, this would make a strong but circumstantial case for upper-airway infections that cause LCR apnea as the mechanism for the increased SIDS incidence in the winter.
Some have speculated that OSA could also cause sudden death in infants because the vast majority of SIDS infants are presumed to die during their sleep and OSA is entirely a sleep-dependent disorder (White, 1995). Indeed, the severity of OSA increases with viral infections of the upper airway, which increase nasal resistance. Moreover, epidemiological studies found that a family history of OSA is a risk factor for SIDS (Tishler et al, 1996) and that brief episodes of OSA were more common in infants who ultimately died of SIDS (Kahn et al, 1992).

$\mathrm{T}$ wo main physiological risk factors for SIDS are heart defects or heart irregularities that, in combination with breathing difficulties, eventually lead to death. One prominent theory proposes that some SIDS infants might have inherent cardiac conduction defects (Schwartz et al, 1998), notably a prolonged so-called QT interval in the heart's electric cycle, which would render the heart susceptible to ventricular fibrillation. Although most patients spontaneously recover from ventricular bradycardia or fibrillation, some can die a sudden death even in the absence of any preceding symptoms (Towbin \& Ackerman, 2001). Several genetic mutations in sodium ion or potassium channels in the myocardial cell membrane cause prolongation of the QT interval (Wang et al, 2007). Of relevance to SIDS, some of these genotypes are associated primarily with death during sleep. The triggering event might be emotional excitement when awake or a sudden stimulus causing arousal from sleep.

There is a large body of evidence to support the idea that a prolonged QT interval has a causal role in SIDS. First, a prolonged QT in the first week of life is strongly associated with SIDS (Schwartz et al, 1998). Second, infants who have died suddenly and unexpectedly have often had a prolonged QT interval before death. Third, $4.3 \%$ of infants who died of SIDS in a large case study had pathological QT mutations (Towbin \& Ackerman, 2001; Wang et al, 2007). All in all, these findings provide strong evidence that a minority of deaths in infants is caused by genetic mutations that prolong the QT interval and cause fatal ventricular bradycardia or fibrillation.

Infants who die from inherited metabolic disorders are technically not SIDS cases. Nevertheless, when an autopsy cannot reveal a clear cause of death, it is often concluded as SIDS. So far, several 
metabolic defects are known to be risk factors for sudden death, such as medium- and long-chain acyl-CoA dehydrogenase deficiency, glutaric acidaemia type 1 and 2, and carnitine palmitoyl transferase type II/ translocation deficiencies. Most infants with these disorders suffer from acute metabolic problems but, when admitted to a hospital, they respond rapidly to medical therapy. Furthermore, most medical examiners now screen for metabolic defects at the post-mortem examination. In the past, approximately $3-6 \%$ of sudden infant deaths were the result of such metabolic disorders (Boles, 2001). However, as postmortem diagnoses are becoming more frequent and accurate, the number of these deaths diagnosed as SIDS is becoming less frequent.

$\mathrm{T}$ he past decade has seen much research into brainstem defects in SIDS infants to explain the failure of the body's own emergency mechanisms to autoresuscitate an infant that has stopped breathing (Kinney et al, 2001; Ozawa \& Okade, 2002). Various studies have identified subtle anatomical changes and receptor deficiencies in brainstem nuclei in infants who have died of SIDS. In many cases, certain nuclei, which are believed to be critical for carbon dioxide sensitivity, arousal from sleep and cardiorespiratory control, were shown to have decreased numbers of serotonergic receptors compared with those in infants dying from other causes. Other studies of SIDS infants have documented inherited defects in serotonin transporter genes (Narita et al, 2001; Maher et al, 2006).

\section{Current theories therefore explain SIDS through a combination of environmental, genetic and metabolic factors that do not cause SIDS themselves, but render an infant more vulnerable}

It seems that most SIDS infants had multiple abnormalities in brain serotonergic systems, which could provide an explanation for why they were more vulnerable to death (Paterson et al, 2006). However, the numbers of neurons that are deficient in serotonin receptors are actually increased in SIDS infants, which might be a compensation for the reduced serotonin receptors on individual neurons (Paterson et al, 2006). As such, these infants might not have abnormal brainstem function. At the current stage of research, it is not clear whether these altered serotonin transporter genes have any pathological significance or represent a possible genetic predisposition to SIDS (Leiter \& Böhm, 2007).

\section{...without solid biological and mechanistic theories [...] it remains difficult to develop reliable diagnostic tests to identify vulnerable children, let alone develop preventive therapies}

Nonetheless, these brainstem abnormalities might explain why autoresuscitation fails when infants reach a critically low level of oxygen saturation in their blood. Further research in this area has concentrated on the possible deficiencies that would prevent arousal from sleep and related carbon dioxide sensory mechanisms.

These efforts are justified by reports that the arcuate nucleus of the brainstem in a few SIDS infants showed hypoplasia-that is, incomplete development (Filiano \& Kinney, 1992). This area of the brain is believed to be a major site of carbon dioxidesensitivity regulation related to respiratory function. However, experiments using antagonists of the specific serotonin receptor sites in the brain have produced only modest decreases in carbon dioxide sensitivity (Curran et al, 2001). Moreover, the reduced carbon dioxide response is seen only in male infants (Pematti et al, 2006). Therefore, as far as the response to increasing levels of carbon dioxide is concerned, it is unclear how a reduction in serotonergic receptors could significantly increase the risk for SIDS. More generally, it is not clear how brainstem serotonergic mechanisms might increase vulnerability to SIDS. In summary, it seems that abnormal serotonergic systems in the brainstem of many SIDS victims could prevent arousal from sleep, or cause alterations in sleep state cycles, cardiovascular control or carbon dioxide responsiveness (Darnall et al, 2005). At present, it remains to be seen how such abnormalities could make infants substantially more vulnerable to SIDS.

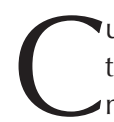

urrent theories therefore explain SIDS through a combination of environmental, genetic and metabolic factors that do not cause SIDS themselves, but render an infant more vulnerable (Guntheroth \& Spiers, 2007). A viral infection, LCR apnea or OSA could trigger an eventually fatal chain of events that start as the child's emergency responses to critical oxygen concentration in the blood fail to kick in.

So far, the greatest progress in preventing the sudden and unexpected death of infants comes from epidemiological research to identify environmental risk factors, and campaigns to educate and advise parents. In particular, the use of lighter bedding and the turning of sleeping infants onto their back have both greatly reduced the number of SIDS cases and accidental suffocation in many countries. However, SIDS is still the second leading cause of early childhood death, after congenital disorders, and the leading cause of death in babies aged between one month and one year. Further progress thus depends on the identification of genetic, metabolic and physiological risk factors, and their exact role in increasing an infant's vulnerability to sudden death. Yet, without solid biological and mechanistic theories of how these various factors combine and act, it remains difficult to develop reliable diagnostic tests to identify vulnerable children, let alone develop preventive therapies. Given the number of infants each year who still die of SIDS, and the traumatic experience that results for their parents, it is a research topic with both a noble goal and potentially huge benefit for society.

\section{REFERENCES}

Abu-Osba YK, Brouillette R, Wilson S (1982) Breathing pattern and transcutaneous oxygen tension during motor activity in preterm infants. Am Rev Respir Dis 125: 382-387

Blair PS, Sidebotham P, Berry PJ, Evans M, Fleming PJ (2006) Major epidemiological changes in sudden infant death syndrome: a 20-year population-based study in the UK. Lancet 367: 314-319

Boles RG et al (2001) Retrospective biochemical screening of fatty acid oxidation disorders in portmortem livers of 418 cases of sudden death in the first year of life. J Pediatr 132: 924-933

Curran AK, Darnall RA, Filiano JJ, Lie A, Nattie

EE (2001) Muscimol dialysis in the rostral ventral medulla reduced the $\mathrm{CO}_{2}$ response in awake and sleeping piglets. J Appl Physio/ 90: 971-980

Darnall RA, Harris MB, Gill WH (2005) Inhibition of serotonergic neurons in the nucleus paragigantocellularis lateralis fragments sleep and decreases rapid eye movement in the piglet: implications for sudden infant death syndrome. J Neurosci 25: 8322-8332 
Davies A, Koenig J, Thach BT (1988) Upper airway chemoreflex responses to saline and water in preterm infants. J App/ Physiol 64:1412-1420

Fagenholz S, Lee J, Downing E (1979) Association of anemia with reduced central respiratory drive in the piglet. Yale J Biol Med 52: 263-270

Filiano JJ, Kinney HC (1992) Arcuate nucleus hypoplasia in the sudden infant death syndrome. J Neuropathol Exp Neurol 51: 394-403

Fleming PJ, Blair PS, Bacon C, Bensley D, Smith I, Taylor E, Berry J, Golding J, Tripp J (1996) Environment of infants during sleep risk of the sudden infant death syndrome: results from 1993-5 case-control study for confidential inquiry into stillbirths and deaths in infancy. $B M$ J 313: 191-195

Guntheroth WG, Spiers PS (2007) The triple risk hypotheses in Sudden Infant Death Syndrome. Pediatrics 110: $\mathrm{e} 64$

Hunt C, Corwin M, Baird T (2004) Collaborative Home Infant Monitoring Evaluation (CHIME) study group. Cardiorespiratory events detected by home memory monitoring and one-year neurodevelopmental outcome. J Pediatr 145: 465-471

Kahn A et al (1992) Sleep and cardiorespiratory characteristics of infant victims of sudden death: a prospective case-control study. Sleep 15: 287-292

Kinney HC, Filiano JJ, White WF (2001) Medullary serotonergic network deficiency in the sudden infant death syndrome: review of a 15 year study of a single dataset. / Neuropathol Exp Neurol 60: 228-247

Leiter JC, Böhm I (2007) Mechanisms of pathogenesis in the Sudden Infant Death Syndrome. Respir Physiol Neurobiol 159: 127-139

Maher BS, Marazita ML, Rand C, Zhou L, Berry-Kravis EM, Weese-Mayer DE (2006) 3' UTR polymorphism of the serotonin transporter gene and Sudden Infant Death Syndrome. haplotype analysis. Am / Med Genet Part A 140A: 1453-1457

Meny RG, Carroll JL, Carbone MT, Kelly DH (1994) Cardiorespiratory recordings from infants dying suddenly and unexpectedly at home. Pediatrics 93: 44-49

Miller MJ, DiFiore JM (1995) A comparison of swallowing during apnea and periodic breathing in premature infants. Pediatr Res 37: 796-799

Mitchell EA et al (1991) Results from the first year of the New Zealand Cot Death Study. N Z Med) 104: $71-76$

Narita N, Narita M, Takashima S, Nakayama M, Nagai T, Okado N (2001) Serotonin transporter gene variation is a risk factor for sudden infant death syndrome in the Japanese population. Pediatrics 107: 690-692

Ozawa Y, Okade N (2002) Alteration of serotonergic receptors in the brainstem of human patients with respiratory disorders. Neuropediatrics 33: 142-149

Paluszynska D, Harris KA, Thach BT (2004) Influence sleep position experience on ability of prone sleeping infants to escape from asphyxiating microenvironments by changing head position. Pediatrics 114: 1634-1639

Patel A, Harris KA, Thach BT (2001) Inspired $\mathrm{CO}_{2}$ and $\mathrm{O}_{2}$ in sleeping infants rebreathing from bedding: relevance for sudden infant death syndrome. J Appl Physio/ 91: 2537-2545

Paterson DS, Trachtenberg FL, Thompson EG, Belliveau RA, Beggs AH, Darnall R, Chadwick AE, Krous HF, Kinney HC (2006) Multiple serontonergic brainstem abnormalities in sudden infant death syndrome. JAMA 296: 2124-2132

Pematti EM et al (2006) Ventilory response to hypercapnia and hypoxia after extensive lesion of medullary serotonergic neurons in newborn conscious piglets. J Appl Physio/ 101: 1177-1188

Pickens DL, Schefft G, Thach BT (1988) Prolonged apnea associated with upper airway protective reflexes in apnea of prematurity. Am Rev Respir Dis 137: 113-118

Pickens DL, Schefft G, Thach BT (1989) Pharyngeal fluid clearance and aspiration preventive mechanisms in sleeping infants. J Appl Physiol 66: 1164-1171

Poets CF, Meny RG, Chobanian MR, Bonofiglo RE (1999) Gasping and other cardiorespiratory patterns during sudden infant deaths. Pediatr Res 45: 350-354

Scheers-Masters J, Schootman M, Thach BT (2003) Heat stress and sudden infant death syndrome incidence a U.S. population based epidemiological study. Pediatrics 113: e586-e592

Schwartz PJ et al (1998) Prolongation of the QT interval and the sudden death syndrome. N Engl J Med 338: 1709-1714
Sridhar R, Thach BT, Kelly DH, Hensley JA (2003) Characterization of successful and failed autoresuscitation in Sudden Infant Death Syndrome (SIDS) and other infants dying sudden and unexpectedly. Pediatr Pulmonol 36: 113-112

Thach BT, Jacobi MS, Gershan WM (1991)

Control of breathing during asphyxia and autoresuscitation. In Developmental Neurobiology of Breathing G Haddad \& JP Farber (eds). New York, NY, USA: Marcel Dekker

Tishler P, Redline S, Ferrette V, Hans MG, Altose MD (1996) The association of sudden unexpected infant death with obstructed sleep apnea. Am J Respir Crit Care Med 153: 1857-1863

Towbin JA, Ackerman MJ (2001) Cardiac sodium channel gene mutations and sudden infant death syndrome. Circulation 104: 1092

Unger B, Kemp JS, Wilkins D, Psara R, Ledbetter T, Graham M, Case M, Thach BT (2003) Racial disparity and modifiable risk factors among infants dying suddenly and unexpectedly. Pediatrics 111: e127-e131

Vege A, Rognum TO (2004) Sudden infant death syndrome, infection and inflammatory responses. FEMS Immunol Med Microbiol 42: 3-10

Wang DW et al (2007) Cardiac sodium channel dysfunction in sudden infant death syndrome. Circulation 115: 368-376

White DP (1995) Sleep-related breathing disorder. 2. Pathophysiology of obstructive sleep apnoea. Thorax 50: 797-804

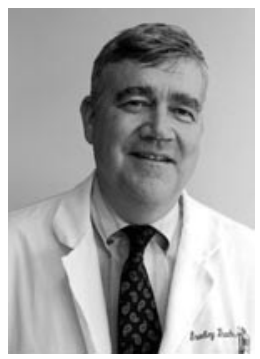

Bradley Thach is Professor of Pediatrics at Washington University in St. Louis, MO, USA. E-mail: thach@kids.wustl.edu

doi:10.1038/sj.embor.7401163 